International Journal of Engineering \& Technology, $7(1.1)(2018)$ 329-332
International Journal of Engineering \& Technology
Website: www.sciencepubco.com/index.php/IJET
Research paper

\title{
An efficient path reconstruction in dynamic and large-scale networks using extensive hashing
}

\author{
David Raju Kuppala ${ }^{1}$, Jayasimha Reddy Ambati ${ }^{2 *}$, Naveen Racharla ${ }^{2}$, Devi Prasanna.K ${ }^{2}$ \\ ${ }^{1}$ Asst. Professor, Koneru Lakshmiah Educational Foundation \\ ${ }^{2}$ Student, Koneru Lakshmiah Educational Foundation \\ *Corresponding author E-mail: jayasimha7777@gmail.com
}

\begin{abstract}
Late remote sensor systems (WSNs) are be-coming logically complex with the creating framework scale and the dynamic thought of remote correspondences. Various estimation and decisive techniques depend upon per-divide courses for correct and fine-grained examination of the psyche boggling net-work hones. In this paper, we propose iPath, a novel way inferring approach to manage reproducing the per-package directing courses in capable and broad scale frameworks. The basic idea of iPath is to abuse high path closeness to iteratively accumulate long courses from short ones. IPath starts with a hidden known game plan of ways and performs way derivation iteratively. iPath consolidates a novel layout of a lightweight Extensible hashing, hash work for affirmation of the construed ways. To furthermore improve the conclusion capacity and moreover the execution capability, iPath fuses a brisk bootstrapping computation to change the hidden game plan of ways. We also execute iPath and survey its execution using takes after from tremendous scale WSN associations and moreover expansive multiplications. Results show that iPath achieves essentially higher revamping extents under different framework settings stood out from other best in class approaches.
\end{abstract}

Keywords: Measurement; Path Reconstruction; Wireless Sensor Networks.

\section{Introduction}

The remote correspondence change is passing on fundamental progressions should data system, telecom, and makes the facilitated frameworks an actuality. Remote framework provide for a overall gathering will recorded regard commitments reporting weight these fast Creating ranges for interest. These gadgets fuse single person electronic accomplices (PDAs), tablets, (PCs), servers [2] .Also printers. Pc gadgets need processors, memory, and an techniques for interfacing for a particular sort of framework.

\subsection{Wireless sensor network (WSN)}

Remote sensor networks (WSN), every so often known as remote sensors Also actuator frameworks (WSAN), are spatially disseminated self-overseeing sensors that screens physical alternately biological conditions, for example, temperature, sound, weight, thus and will helpfully pasquinade their majority of the data through those framework should an essential territory [5]. The forefront frameworks need aid bi-directional, and it empowers control about sensor development. Those change from claiming remote sensor frameworks might have been propelled by military provisions similar to bleeding edge surveillance. Presently An day's such sort frameworks need aid used Similarly as An and only various mechanical Also customer applications[1], Concerning illustration up to date methodology checking Also control, machine wellbeing observing, thus. Those WSN may be created for amount for hubs that extents starting with several to a couple hundreds or Indeed thousands, the place each center will be connected with An sensor. Each sensor a center need a couple areas like: An radio handset with internal gathering apparatus, microcontroller, an electronic circlet which will be used to interfacing the sensors What's more An vitality hotspot [2]. A sensor center might vary done measure starting with that of a compartment gauge down of the degree of a grain for tidy, albeit working odds from claiming veritable moment estimations at present can't appear to be should a chance to be made. The expense from claiming sensor hubs is similarly factor, Also ranges starting with several to hundred dollars, dependent upon the diverse nature from claiming each singular sensor hubs. Measure Furthermore expense necessities ahead sensor hubs acquires around relating imperatives with respect to assets, for example, vitality, memory, pace and information transmission[9].

\section{Related works}

In wired ip systems, fine-grained organize estimation incorporates various angles, to example, controlling best approach recreation, pack postpone estimation, Also package adversity tomorrow. Done these works, tests would used for estimation reason. Traceroute may be an conventional framework systematic gadget for indicating the manner various tests. DTrack will be an test built route Emulating schema that predicts What's more tracks web manner transforms. Likewise shown toward those desire for manner changes, D Track might track route transforms viably [7]. Fine Comb is a current test based framework deferral and setback geology approach that spotlights around settling package rearrangement. To a chance to be told, a present worth of effort layouts the arrange space of looking at calculations to organize execution estimation. Using tests, be that concerning illustration it may, will be typically not alluring done WSNs. Those grade reason may be 
that the remote changing may be troublesome on be got Eventually Tom's perusing few tests, Also constant looking at will exhibit secondary vitality usage. An current worth of effort researches those issue from claiming recognizing per-jump estimations starting with end-to-end best approach estimations, under those supposition that association estimations would included substance What's more unfaltering. Without using any progressive test, it develops a straight skeleton by the endto-end estimations from Different internal screens. Path information will be needed with exist as prior majority of the data with fabricate the regulate skeleton[3] .Thusly, this worth of effort will be orthogonal to iPath, What's more combining them might prompt new estimation techniques to WSNs. There are a couple late best approach revamping methodologies to WSNs. Pad will be An decisive instrument flying that incorporates An pack stamping arrangement to secure those framework toponomy. Pad acknowledge a reasonably static framework Furthermore employments each pack should pass on you quit offering on that one ricochet of a manner. During the perspective at the framework finishes dependent upon evidently powerful, the consistently evolving administering manner can't make decisively recreated. MNT to start with gets an course of action from claiming robust packs from those got parcels during sink, at that perspective uses those trustworthy package situated should imitate each got parcel's way [4]. The point when the framework isn't greatly interesting and the package movement extent will be high, MNT camwood finish helter skelter amusement extent for helter skelter propagation cost precision. Nonetheless, similarly as depicted done segment V-C, MNT is vulnerableto pack setback What's more remote components. PathZip hashes those Steering lifestyle under a 8-B hash a impetus to each package. In that point, the sink assumes out an careful search through those neighboring hubs to An match. The issue about PathZip may be that the pursuit space develops fast at the framework scales up. Pathfinder anticipate that all hubs transform neighborhood parcels Also need an ordinary interpacket between time (i. E., IPI). Pathfinder uses the transient relationship between's Different package approaches What's more adequately packs the path information under each pack. During that point, in the pc side, it could infer pack routes from those compacted information. Contrasted with PathZip, iPath abuses secondary best approach likeness the middle of diverse packs to fast surmising, bringing something like a significant part preferred versatility [4-5] .Contrasted for MNT, iPath need Extensively lesquerella stringent necessities with respect to productive route induction: clinched alongside each jump, iPath recently obliges no less one neighborhood package Emulating An comparative way, same time MNT obliges a course of action for constant packs for An comparable guardian (called trustworthy parcels). Contrasted for Pathfinder, iPath doesn't hope standard IPI. IPath accomplishes higher diversion proportion/precision in distinctive framework states by misusing path closeness "around routes with Different lengths.

\section{Problem statement}

\subsection{Existing model}

The mossycup oak existing deferral and setback estimation methodologies anticipate that those controlling toponomy may be provided for similarly as from the prior. Those time-fluctuating Steering toponomy might make effectively gotten toward per-parcel controlling way, in a far-reaching way upgrading the profits of existing WSN postponement and adversity tomorrow methodologies. An acceptable approach will be with associate those entire guiding possibility to get to each package. The issue from claiming this approach is that its message overhead camwood be enormous to parcels with in length controlling approaches. Recognizing the compelled correspondence holdings for WSNs, this approach is typically not engaging before long.

\subsection{Proposed system}

We recommend iPath, a novel manner incitement approach will manage reproduce running routes at those sink side. For light of a real mind boggling urban identifying framework with the sum center making close-by bundles, we spot a magic perception: it may be altogether liable that a package starting with center Furthermore a standout amongst those parcels from 's guardian will take after a comparable best approach starting starting with 's guardian at the sink. We imply with this discernment likewise secondary manner likeness. IPath accomplishes a significantly higher diversion extent to frameworks with reasonably low package movement extent Furthermore helter skelter guiding stream. The commitments of this fill in need aid the going with. We watch helter skelter best approach similarity clinched alongside a correct sensor c. In perspective of this perception, we recommend an iterative boosting computation to proficient path induction. We recommend a lightweight hash fill in for profitable check inside iPath. We also recommend an fast bootstrapping computation with improve those incitement limit and What's more its execution proficiency. We recommend an explanatory model to figure those productive revamping probability in distinctive framework conditions, to example, organize scale, controlling elements, package misfortunes, Furthermore center thickness. We execute iPath Furthermore evaluate its execution using takes after starting with broad scale WSN associations Also also expansive reproductions. IPath accomplishes higher propagation cost extent under Different framework settings contrasted for states of the workmanship.

\section{Ipath design}

Those want of iPath incorporates three sections: iterative boosting, PSP-Hashing, Furthermore fast bootstrapping. Those iterative boosting count may be the key bit for iPath. It uses those short approaches will imitate long routes iteratively in perspective of the lifestyle similarity. PSP-Hashing provides for an approach likeness ensuring hash worth of effort that makes the iterative boosting figuring have those ability with weigh if two routes need aid similar with secondary precision [6] .During the purpose The point when those around the world period chance and the guardian change counter would joined under each parcel, a fast bootstrapping technique will be also used to quicken the iterative boosting count What's more What's more should change that's only the tip of the iceberg routes. An. Iterative boosting iPath recreates dark long approaches from known short approaches iteratively. Eventually Tom's perusing taking a gander at the recorded hash regard and the determined hash esteem, those sink could weigh if An in length approach Also a short path offer An comparable path following those short way's exceptional center. During those perspective the point when the sink figures a match, the in length manner could be remade Eventually Tom's perusing combining its interesting center and the short path. B. B. PSP-Hashing Concerning illustration specified in the iterative boosting calculation, those PSPHashing (i. E., best approach likeness saving) expects An magic Some piece to impact those sink on bring those limit on check if a short manner will be similar with in turn in length approach. There would three necessities of the hash fill.Those hash limit ought on a chance to be lightweight What's more sufficiently successful since it ought be stay with running for benefit urged sensor hubs. The hash limit ought will a chance to be organize fragile. That is, hash (A, B) Also hash (B, An) ought not be the same. The crash probability ought should a chance to be enough low will Fabricate the propagation cost precision. C's. Fast Bootstrapping those iterative boosting computation needs an underlying course of action of recreated routes. Regardless those one/twobounce ways, the fast bootstrapping figuring also provides for All the more initial reproduced approaches of the iterative boosting calculation. These introductory recreated ways lessen the amount of iterations required and accelerate those iterative boosting calculation. 


\section{Extensible hashing}

\subsection{Simple hashing}

An champion "around those A large portion troublesomeness things done get ready is securing something the place you could find it once more. The mossycup oak clear framework is on store the information clinched alongside composed request and gaze utilizing An twofold yield for sample[8] .Hashing will be a fundamentally that's only the tip of the iceberg energetic method to finishing On in way fill in. Every one you require will be a suitableness hashing cutoff.

\subsection{HASH (k)}

Say which will make the information see k, the key, Also change over it under an breaking point range.

The information is after that secured toward the extend What's more assuming that you ever require it once more you fundamentally utilize HASH (k) on Figure it. For example, on keep an outline of names What's more addresses keyed on surname you might utilize an hash worth of effort which joined the ascii estimations about every leto in the surname. Those coming about aggravator Might At that point a chance to be utilized concerning illustration those record should a combination of records used to store the name and location.

When you require the record once more you basically hash the surname What's more try straight of the zone the place it will be secured. The grade multifaceted outline with hashing may be that hash breaking points are harmed and every now and again send arranged keys will a similar extent. For example, two zones with the arranged surnames may be mapped to a similar show fragment [7] .This will be known as An crash and hashing frameworks contrast in the best approach that they conform of the issue. Open hashing barely chains the influenced things off those person presentation zone something like that sensibly securing them constantly on In a similar one assembly record a stimulus Similarly as An straight rundown. End hashing employments an hashing cutoff for admiration to each moment chance to provide for another area in the presentation the place the thing that brought about the crash could a chance to be secure.

\subsection{Extensible hashing}

The usage of a square table to depict bits of the hash being utilized to the limit pieces is the key idea. You can reuse ruins until the point that they are full. Pieces simply should be part when they finish off.

Clearly notwithstanding you have to look the piece for the right a motivating force in which you are charmed, yet this isn't a honest to goodness overhead. If the squares are coordinated to be units of plate amassing then you are still guaranteed to get to the correct piece, i.e. the one that contains the data you are scanning for, in a single circle read. Once the piece is in memory it can be looked quickly despite using a fundamental straight chase. The fundamental bona fide overhead is the need to keep a rundown table that augmentations by a vitality of two each time another bit of the hash regard is used to extend the amount of squares, however again a little math exhibits this too isn't an issue as long as the square size is sensibly generous. hash result.

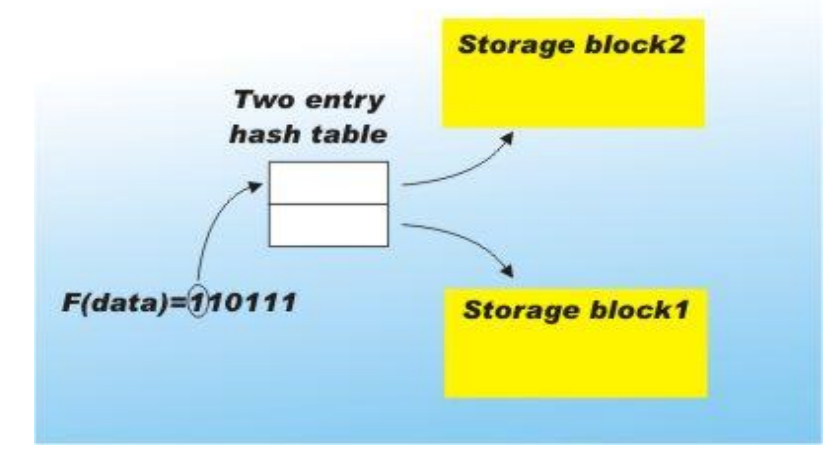

You might perceive that using this course of action all that acknowledged bit of the way regards would secured clinched alongside each bit.

A little while later think about what happens at a standout amongst the squares winds dependent upon continuously full. Those undeniable improvemen is expand the measure of squares connected with the hashing by Creating those measure of the record table.In the occasion that we use another bit of the hash appreciation the explore of the table duplicates What's more it could oblige twofold those aggravator amount about squares.

This Creating utilization of those odds provided for toward the hash fill in may be those relic that makes the skeleton extensible.

The essential issue that remarkable parts may be that we necessity with repair those introduce ends for the target that those full squares are spilt under two new squares holding the fluctuating estimations of the second bit of the hash deference. Every of the full squares could make some piece under two bits by on a general feeling using the estimations of the extra hash spot. That is whether the full square held every single a standout amongst those majority of the data that hashed with 1 say At that point this bit might a chance to be part under each single a standout amongst the data that hashes will 10 Also 11.

The splendid thing will be that we don't require will do anything of the keeps that need aid not full [13] .Those square table might in a far-reaching way organization estimations of the beginning late used hash spot on a comparative bit. To instance, if those bit that holds data hashed to 0 isn't full the table recently maps 00 Furthermore 01 will an commensurate square.

Good when an square that need rehashed ranges in the table finally complete off it could a chance to be a component in the commonplace best approach.

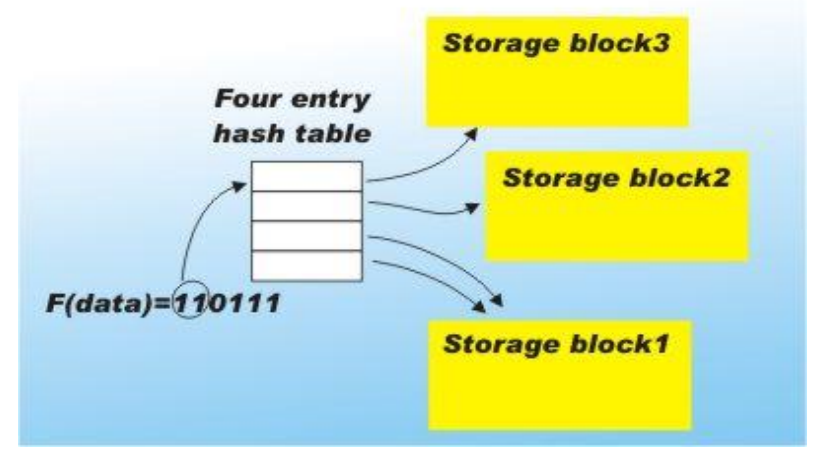

Those utilization of a square table should portray odds of the hash constantly used as far as possible ends is those way thought. You might reuse vestiges until those purpose that they are full. Ends essentially ought to make part the point when they complete off. Obviously Despite you must look the bit for the correct a inspiring power to which you need aid charmed, yet this isn't An fair on goodness overhead. Though the squares need aid facilitated with make units about plate amassing then you would still guaranteed to get of the right piece. The particular case that holds those information you need aid filtering for, on An absolute circis siliquastrum read. 
Once those bit will be On memory it might a chance to be gazed fast in spite of utilizing an essential straight pursue. Those basic true blue overhead may be those necessity on keep An rundown table that augmentations by a vitality about two each duration of the time an additional spot of the hash respect will be used to augment the measure about squares, Notwithstanding once more a minimal math exhibits this a really isn't an issue as in length as those square size will be sensibly liberal.

\section{Results}

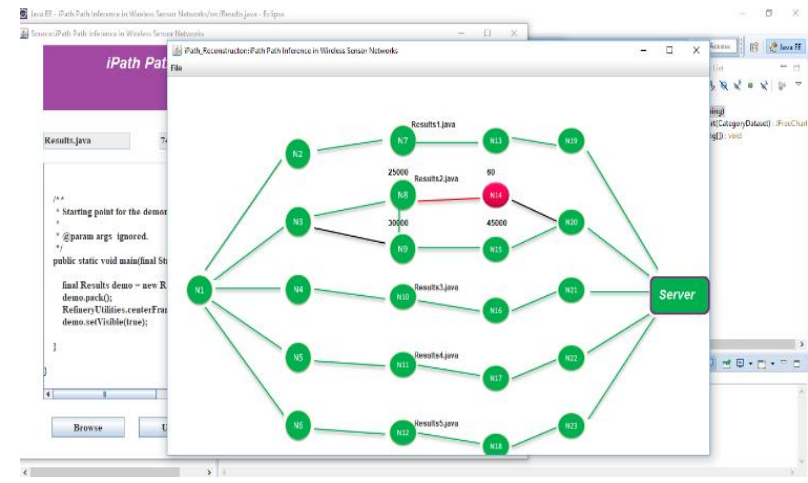

\subsection{The architecture of the proposed solution}

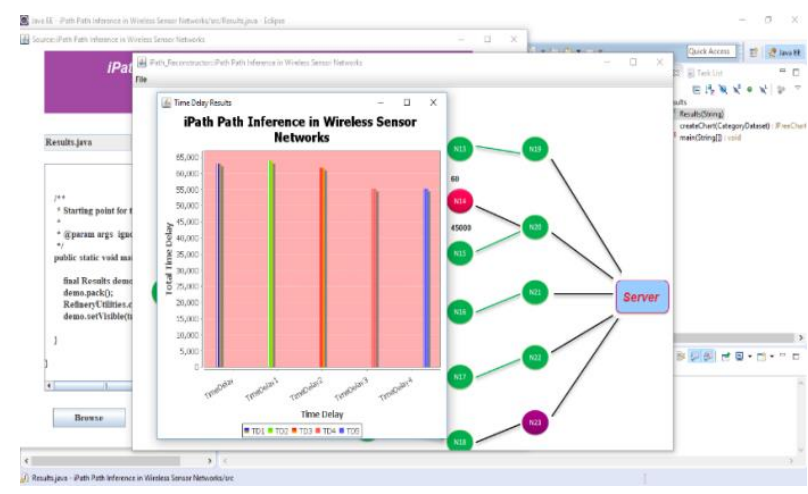

6.2. The results observed in the proposed process

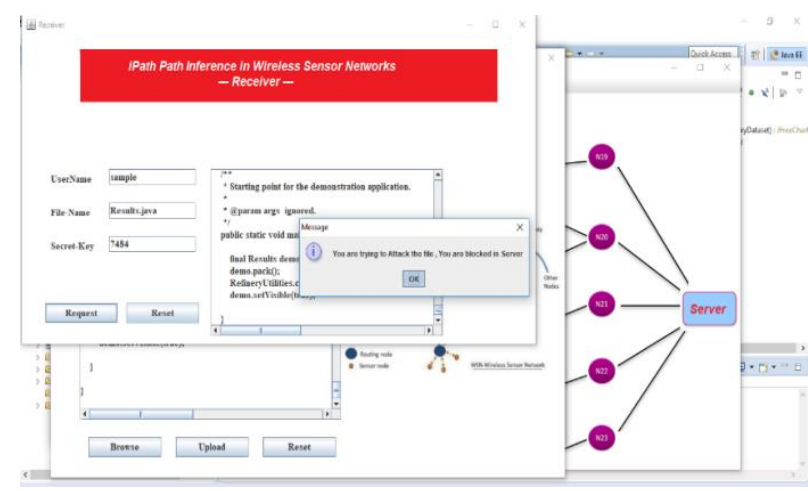

6.3. The added security process blocking the attackers validating the secret key.

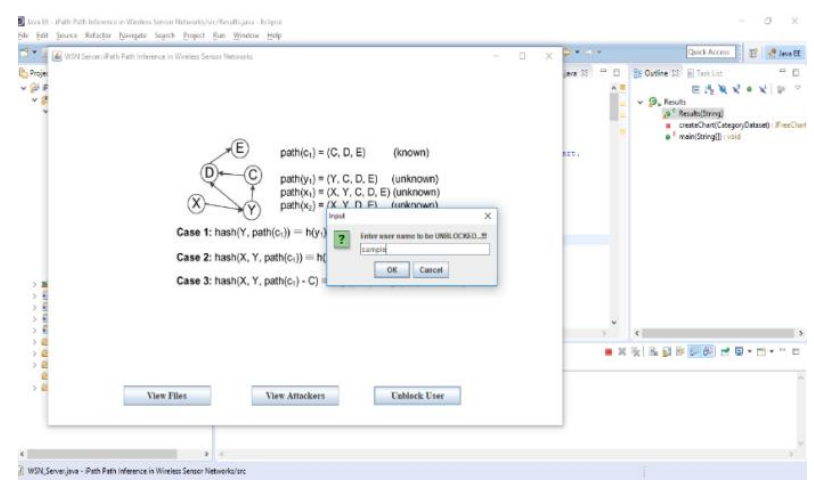

6.4. The unblocking process form admin's end

\section{Conclusion}

In this paper, we recommend iPath, a novel way induction methodology to reconstructing the directing way for each gained bundle. IPath exploits the way similitude Furthermore utilization those iterative boosting algorithm on recreate those steering way successfully. Furthermore, those quick bootstrapping algorithm gives a starting set from claiming ways for the iterative algorithm. We formally dissect the remaking execution from claiming iPath and in addition two related methodologies. The Investigation Outcomes indicate that iPath accomplishes higher remaking proportion at the organize setting varies. We also execute iPath Furthermore assess its execution Eventually Tom's perusing an tracedriven contemplate Furthermore broad simulations. Contrasted with states of the art, iPath accomplishes substantially higher reproduction proportion under distinctive organize settings.

\section{References}

[1] M. Ceriotti et al., "Monitoring heritage buildings with wireless sensor networks: The Torre Aquila deployment," in Proc. IPSN, 2009 pp. $277-288$.

[2] L. Mo et al., "Canopy closure estimates with GreenOrbs: Sustainable sensing in the forest," in Proc. SenSys, 2009, pp. 99-112. https://doi.org/10.1145/1644038.1644049.

[3] X. Mao et al., "CitySee: Urban CO2 monitoring with sensors," in Proc. IEEE INFOCOM, 2012, pp. 1611-1619.

[4] O. Gnawali, R. Fonseca, K. Jamieson, D. Moss, and P. Levis, "Collectiontree protocol," in Proc. SenSys, 2009, pp. 1-14.

[5] D. S. J. D. Couto, D. Aguayo, J. Bicket, and R. Morris, "A highthroughputpath metric for multi-hop wireless routing," in Proc. MobiCom,2003, pp. 134-146.

[6] Z. Li, M. Li, J. Wang, and Z. Cao, "Ubiquitous data collection formobile users in wireless sensor networks," in Proc. IEEE INFOCOM,2011, pp. 2246-2254.

[7] X. Lu, D. Dong, Y. Liu, X. Liao, and L. Shanshan, "PathZip: Packetpath tracing in wireless sensor networks," in Proc. IEEE MASS, 2012, pp. 380-388. https://doi.org/10.1109/MASS.2012.6502538.

[8] M. Keller, J. Beutel, and L. Thiele, "How was your journey? Uncoveringrouting dynamics in deployed sensor networks with multihopnetwork tomography," in Proc. SenSys, 2012, pp. 15-28.

[9] Y. Yang, Y. Xu, X. Li, and C. Chen, "A loss inference algorithm forwireless sensor networks to improve data reliability of digital ecosystems., "IEEE Trans. Ind. Electron., vol. 58, no. 6, pp. 21262137, Jun.2011. https://doi.org/10.1109/TIE.2011.2106096. 